\title{
Identité, Légitimité et Rhizome dans Les deux fins d'Orimita Karabegovic de Janine Matillon.
}

\section{Muriel PLACET-KOUASSI}

ui suis-je? Qui sommes-nous? Ces questions
identitaires, posées de tout temps, font l'objet de débats infinis
à différents niveaux: individuel, communautaire, national,
culturel, etc.

La notion d'identité détermine ou informe les relations entre individus et surtout entre peuples. C'est elle aussi qui sert de prétexte aux guerres, aux conquêtes territoriales, aux massacres génocidaires et autres.

Affirmer son identité, cela a souvent voulu dire rechercher une légitimité dans et par ses racines, dans et par sa généalogie ou sa filiation. Nous nous proposons donc ici d'analyser dans le roman de Janine Matillon, Les deux fins d'Orimita Karabegovic ${ }^{1}$, dans quelle mesure la notion d'identité fondée sur une légitimité généalogique fait violence puisqu'elle implique nécessairement l'exclusion de l'autre. Il nous semble que le processus de légitimation de l'individu fondée sur une identité qui serait donnée par sa lignée (ses origines et sa filiation) est ce qui rend possible (voire, ce qui mène à) des actes aussi destructeurs que le génocide et la purification ethnique qui impliquent la violence, la négation forcée de l'autre, alors que le concept de l'être (ou plutôt de l'étant) dont l'identité serait de l'ordre du multiple, en devenir et en relation avec son entour, rend caduque l'idée d'une légitimation fondée uniquememt sur l'identité et l'origine généalogique de l'individu. 
Si la notion d'identité est problématique pour l'individu, elle l'est tout autant pour la communauté ou la nation. Ainsi nous assistons ces quelques dernières années en France à de sérieuses querelles au sein de l'Ined, 1'Institut National d'Études démographiques. La démographie se donne pour objet de décrire, d'étudier et de dénombrer un peuple ou une population donné(e) en vue d'établir les taux de natalité et de mortalité ou d'analyser une population par sections d'âge ou de profession par exemple. Encore faudrait-il être en mesure de définir par avance quelle population fera l'objet d'une enquête démographique. Et c'est là que le bât blesse au sein des démographes de l'Ined qui ne peuvent s'accorder sur les critères qui font aujourd'hui qu'un français est français. Ainsi, Hervé Le Bras (chercheur à l'Ined) repproche à l'une des ses collègues, Michèle Tribalat, d'avoir au cours d'une enquête démographique introduit la notion d'origine ethnique comme critère de sélection d'appartenance à la population française. Légalement, la nationalité est le seul critère reconnu qui décide si tel ou tel individu est français. Dans l'enquête en question, l'enquêtrice interroge ses interlocuteurs sur deux critères problématiques que sont "l'appartenance ethnique" fondée sur la langue maternelle et "l'origine ethnique" définie par le lieu de naissance des parents. Il est facile d'anticiper les problèmes qui découleront de ce choix de critères quand on connaît l'hétérogènéité de nombreux foyers français Derrière des prétentions scientifiques, il semble que cette démarche veut redonner un sens à cette fameuse expression "français de souche", notion qui n'est rien d'autre qu'un fantasme ou voeu de hiérarchisation des populations au sein d'une nation.

Le texte qui nous intéresșe ici ferait plutôt allusion à la notion de "serbe de souche" (ou "slave de souche") dans l'ex-Yougoslavie. Ce roman de Janine Matillon, publié en 1996 aux Éditions Maurice Nadeau et intitutlé Les deux fins d'Orimita Karabegovic, raconte l'histoire tragique du personnage éponyme, une jeune femme bosniaque, emprisonnée par les Serbes qui ont décidé de "purifier" leurs prisonnières en les ensemançant.

Ce livre pénètre par touches effroyables dans les méandres de ce procédé eugénique utilisé par un peuple sur un autre, à savoir la purification ethnique. Il ne s'agit pas ici d'un génocide à proprement parler, c'est-à-dire d'un acte visant à l'élimination totale d'une "race" ou d'un groupe ethnique, un acte visant à remplacer une présence par une absence. Le roman de Matillon explore plutôt le procédé eugénique qui relève d'une transformation forcée, d'une métamorphose douloureuse. D'un côté, il y a arrêt brutal (dans le cas du génocide), de l'autre, il y a 
passage forcé (dans le cas de la purification ethnique). Ce roman donne à lire le processus de résistance d'un être (Orimita) au moment même où son identité est en crise parce que menacée dans son fondement et dans son devenir, c'est-à-dire niée dans ses origines généalogiques et remise en question dans sa filiation à venir.

Orimita Karabegovic est une jeune femme bosniaque, née de père musulman et de mère croate. Dans un premier temps, les origines paternelles musulmanes d'Orimita seront qualifiées de "regrettables" et réduites à un "accident génétique" par ses geôliers serbes. En vue de corriger ces erreurs originelles, les Serbes ensemanceront ces matrices musulmanes ou croates pour créer une légitimité serbe érigée en valeur fondatrice. Le personnage d'Orimita luttera jusqu'au bout en tant que femme, en tant que bosniaque, en tant que musulmane, et en tant qu'intellectuelle: elle contrecarre les projets purificateurs des Serbes en n'enfantant pas (ou presque) et en refusant cette nouvelle identité (rétroactive) que les Serbes forcent sur elle et en elle dans un souci d'eugénisme idéologique.

Pour analyser la notion d'identité qui sous-tend l'ensemble du roman, nous voudrions dans un premier temps rappeler la distinction que fait l'écrivain-philosophe martiniquais, Édouard Glissant, entre d'un côté "l'identité-racine" et de l'autre "l'identité-relation" (notamment dans son essai Poétique de la Relation ${ }^{2}$ ). L'idée de l'identité-racine serait conçue à partir de la croyance au mythe de la création du monde, épisode fondateur qui donnerait tout son sens à une légitimité de la filiation ainsi qu'à une légitimité de la possession du territoire par une communauté. Alors que l'identité-relation serait donnée dans le vécu des contacts des cultures, hors de toute recherche légitimante ou de conquêtes territoriales, dans ce que Glissant appelle "la trame chaotique de la Relation et non dans la violence cachée de la filiation"3.

Dans le roman de Matillon, il n'est pas donné au lecteur de remonter jusqu'à cet épisode mythique de la création du monde comme le fait Marguerite Yourcenar dans son texte autobiographique Archives du Nord où elle re-crée le début des temps lorsque l'homme n'existait pas encore pour découvrir comment le monde et l'histoire du monde ont accouché d'elle, démarche plus individuelle que communautaire qui échoue puisque la narratrice ne réussit pas à raccorder l'épisode de la Genèse à sa propre filiation ancestrale.

Qu'il s'agisse d'une tentative de remonter sa lignée jusqu'à ce moment fondateur de la Genèse ou bien qu'il s'agisse, comme c'est le cas dans Les deux fins d'Orimita Karabegovic, pour les gêôliers serbes de rappeler 
les évènements historiques déterminants du XIVème siècle, il est clair que nous avons toujours affaire à un passé érigé en valeur fondatrice et légitimatrice du présent. Car c'est en 1389 que les Turcs ont conquis les territoires appartenant aux Serbes. Pour donner une certaine légitimité à la violence, à la guerre, aux tentatives de lavage de cerveau et au viol systématique de ces femmes non-serbes, le personnage qu'on appelle le Professeur (qui est en fait le maître-exécuteur de ce grand programme expérimental que subissent les prisonnières), sollicite le caractère sacré d'un passé qui légitimerait des actes barbares actuels:

Le président de la République de Serbie appelait bien son peuple à se battre jusqu'à la dernière goutte de son sang pour retrouver ses frontières historiques, la terre sacrée de ses ancêtres, son honneur bafoué au XIVè siècle. (19)

Les Serbes ne faisaient que reprendre une terre qui était la leur avant la victoire turque de 1389. Ils renouaient avec le XIVe siècle. (150)

Ainsi, la notion d'identité est ici en prise directe avec l'idée de possession du territoire plus que d'appartenance au territoire. Comme le dit l'écrivain martiniquais, l'identité, "cette revendication ou cette connaissance d'une lignée inscrite dans un territoire [.. .] se gagnera quand les communautés auront tenté, par le mythe ou la parole révélée, de légitimer leur droit à cette possession du territoire." 4

L'histoire de ce roman s'articule autour du concept de territoire, de conquête du territoire qui serait soit l'effet soit la cause d'une affirmation identitaire. L'auteur ne s'attache pas à relater textuellement la conquête territoriale des Serbes, mais en exposant l'ensemencement de ces femmes bosniaques par leurs geôliers serbes, il explore le concept d'une identité qui découlerait d'un processus d'identification à un peuple, à une communauté voire à une nation, processus lui-même lié à la notion de possession ou de conquêtes territoriales.

Ici, c'est bien la femme qui tient lieu de terre à conquérir. Les conquérants se donnent pour mission (en fait une expérience de laboratoire) d'investir les méandres de leur cerveau et les profondeurs de leur matrice. La profession de foi du Professeur n'est-elle pas “toute terre habitée par un serbe est une terre serbe, j'en ajoute une autre: toute matrice fécondée par un Serbe est une matrice serbe" (71). Une telle revendication présuppose que l'homme est le seul garant et continuateur 
de sa lignée, ce qui exclut l'Autre (en l'occurrence la femme) qui représente la terre que la conquête transformera en territoire pour reprendre la pensée de Glissant: "Le territoire est une base pour la conquête. Le territoire exige qu' on y plante et légitime la filiation. Le territoire se définit par ses limites, qu'il faut étendre. Une terre est sans limites, désormais"s. Cette idée d'une conquête territoriale qui passerait par une conquête de la maternité des femmes n'est certes pas une invention serbe. Les viols sur les femmes dans des situations de guerre sont courants. Même si certains de ces viols sont imputables à une violence individuelle spontanée dans le contexte chaotique de la guerre, il s'agit aussi souvent, et c'est le cas ici, d'une démarche collective délibérée qui aurait pour objectif de prolonger, de re-créer ou de fonder à nouveau et ailleurs une filiation dont l'homme serait l'unique garant.

L'identité-racine fait violence dans la mesure où elle nie l'existence de l'autre. L'identité-racine n'est concevable que si un individu établit son identité par rapport à une filiation, à une ascendance en ligne droite qui lui permettrait de trouver une légitimité en remontant directement jusqu'à (idéalement) la création du monde. Une filiation en ligne droite présuppose la négation de nombreux éléments généalogiques. En effet, si j'entreprends de remonter mon ascendance, je dois partir de moi-même pour arriver à deux éléments (mes parents) puis à quatre éléments (mes grands-parents) et ainsi de suite avant d'être pris de vertige devant cette multitude d'ancêtres qu'il me sera difficile de compresser et de raccorder au premier couple mythique (ou biblique). De même, et à l'inverse, si je considère le premier couple mythique comme point de départ d'une descendance dont je serai le point d'arrivée, il me sera impossible d'arriver à moi-même en ligne directe sans éliminer ou nier de nombreux éléments de ce schéma généalogique arborescent. Il est intéressant de noter qu'Orimita ne revendique que son ascendance paternelle (elle se dit musulmane) et passe sous un silence révélateur son origine croate (maternelle). C'est donc par l'homme exclusivement qu'on remonterait aux sources; la femme, elle, ne serait que secondaire mais pourtant vitale en ce sens qu'elle tient lieu de terre, de terreau qui reçoit la semence de l'homme, seul véritable porteur et continuateur de la lignée. La légitimité passe par l'adhésion ou par la croyance à la transparence - celle de la filiation directe qui remonterait aux origines, par exemple - mais lorsque le linéaire est subverti par l'idée d'étendue ou de totalité, la transparence se brouille, et la légitimité est remise en question.

Orimita porte en elle toutes les contradictions d'une communauté qui recherche une légitimité dans une filiation qui remonterait au plus 
près de la Genèse et qui par là même meurt d'asphyxie en se refermant sur elle-même. Par exemple, quand Orimita s'apprête à passer sa première nuit dans le poulailler (ainsi appelle-t-on ce camp de prisonnières), elle s'empêtre dans sa chemise dont la largeur n'est qu'un indice de plus sur cette maternité imposée, cette fécondation à venir. Cette chemise de nuit est "beaucoup trop vaste pour son corps, beaucoup trop vaste pour n'importe quel corps de femme" (39). L'idée de territoire implique des limites -par opposition à l'étendue sans limite de la terre. La femme est, ici, une terre que la semence serbe va conquérir en vue d'une légitimation identitaire et territoriale. La maternité (symbolisée par la chemise de nuit) est cette terre ouverte, le lieu illimité de possibilités illimitées, l'espace du continuum en étendue, l'espace de la Relation.

Orimita refuse cette maternité comme espace de la Relation. Dans un premier temps, son refus est compréhensible dans le contexte des événements du conflit. Orimita n'a pas consenti à cette maternité, elle est victime d'une série de viols systématiques. Toutefois, son refus va plus loin dans la mesure où elle rejette toute idée de métissage, de mélange contre-nature tant l'idée de l'identité-racine informe sa relation au monde, son être au monde. Une fois fécondée par les Serbes, Orimita refusera toujours de se référer à l'être qu'elle attend si ce n'est en terme de kyste, de tumeur maligne, de maladie. Un jour, elle

eut la vision d'une école dont elle était l'institutrice. Elle avait assis devant elle, sagement, les bras croisés, de pauvres, pauvres petits monstres, difformes et débiles et qui ignoraient qu'ils étaient le fruit d'accouplements contre-nature, entre un esprit dément et un corps disgracié, entre des guerriers poilus et sanglants et des filles à demi folles. (128)

En fait, il nous semble que la nature s'arrange fort de ces accouplements. C'est Orimita, ici, qui ne peut concevoir de tels accouplements. Elle ne peut les concevoir mentalement, pas plus que physiquement d'ailleurs. Ainsi, après avoir accouché, alors qu'elle se prépare à tuer "le produit de sa conversion matricielle", elle se rend compte qu'elle a enfanté un mortné et se prend bien tard d'une certaine tendresse pour "ce petit bâtard d'un Serbe et d'une Musulmane qui avait eu la sagesse de mourir avant de naître, à seule fin d'éviter un crime à sa mère" (162). S'il avait (sur)vécu, cet enfant aurait été l'agent de perversion de la filiation. La chaîne de la filiation aurait été dissoute à jamais dans l'étendue nouvelle pour reprendre un vocabulaire glissantien. 
Dans un chapitre de la Poétique de la Relation, Edouard Glissant analyse les mythes fondateurs occidentaux. Le monde occidental - en vue d'assurer la légitimité de son pouvoir sur le reste du monde - a toujours cherché à inscrire sa généalogie dans une projection linéaire qui retracerait l'histoire de l'Occident ou de telle communauté depuis le présent jusqu'à cet acte de la création. Quand le bien-fondé du pouvoir ou de la possession du territoire par un peuple ou une nation est remis en question, on fait presque toujours appel à la filiation pour remonter jusqu'au plus près de la Création pour déterminer qui étaient les premiers occupants du territoire en question. Le monde occidental se pense en terme d'Histoire. À cette pensée de la linéarité et de la chronologie qui rassure une communauté quand à la légitimité de son pouvoir, nous opposerons à l'instar de Glissant, la Relation.

La Relation, c'est en quelque sorte, l'énergie poétique du monde, du monde conçu comme une totalité non-généralisante, une totalité où les singularités remplacent les différences, où les histoires remplacent l'Histoire, où l'étendue remplace la filiation, où le rhizome remplace la racine, où l'identité-relation remplace l'identité-racine. C'est la pensée du Divers (du multiple) opposée à la pensée de l'Un (de l'unique). Penser le multiple, ce n'est pas ajouter des éléments les uns aux autres pour construire un ensemble déterminé, c'est plutôt extraire un à un des éléments de la totalité comme l'entendent Deleuze et Guattari dans leur ouvrage commun Mille Plateaux:

Le multiple, il faut le faire, non pas en ajoutant toujours une dimension supérieure, mais au contraire le plus simplement, à force de sobriété, au niveau des dimensions dont on dispose, toujours n-1 (c'est seulement ainsi que l'un fait partie du multiple, en étant toujours soustrait). Soustraire l'unique de la multiplicité à constituer; écrire à n-1. Un tel système pourrait être nommé rhizome. ${ }^{6}$

Et c'est bien de rhizome qu'il s'agit dans l'espace de la Relation où l'idée de la racine unique est inexistante mais où la notion d'enracinement donne à penser une racine démultipliée en réseaux qui n'aurait ni centre ni périphérie. La pensée du rhizome serait à l'oeuvre dans une poétique de la Relation, selon laquelle, pour reprendre les termes de Glissant "toute identité s'étend dans un rapport à l'Autre."

Dans le roman qui nous intéresse, le tragique provient de ce que les différentes communautés concernées ne réussissent pas à vivre la Relation 
pleinement. Le contact des cultures et des spécificités devient une guerre des différences et de l'exclusivité; le chaos-monde doit être ordonné, hiérarchisé, l'étendue nouvelle doit être délimitée en territoire possédé. La conception rhizomatique du monde invalide la notion d'exclusion dans la mesure où le monde est pensé comme totalité. Et la totalité par définition ignore l'exclusion. Penser le monde, la totalité-monde, ce que Glissant nomme le chaos-monde, c'est soustraire la spécificité de l'agencement rhizomatique, c'est écrire à n-1. C'est parce qu'Orimita se pose en différence totale qu'elle ne peut concevoir mentalement pas plus que physiquement l'intrusion du sang serbe dans sa descendance. C'est parce qu'elle souscrit à la pensée de l'Un qu'elle refuse de participer à l'énergie du chaosmonde. C'est aussi parce que le Professeur serbe souscrit à cette même pensée de l'Un, qu'il se donne pour objectif d'assimiler Orimita et ses congénères.

La filiation garantit la légitimité d'un peuple, elle rassure la communauté du bien-fondé de son pouvoir sur un autre peuple ou de son bon-droit à la possession du territoire. Mais dans de nombreux endroits de la planète et de tous temps, des peuples ou des nation se battent quand il y a trop d'Histoire et pas assez de géographie. Alors si la Relation est refusée, si le "ou" exclusif l'emporte sur le "et" qui permet la coexistence des singularités, l'Autre doit être assimilé ou bien détruit. Orimita choisit la destruction, le Professeur, lui, choisit l'assimilation: "À l'élimination du non-serbe, il préférait sa conversion [...]. Une conversion sur la base de l'esprit. Matricielle d'abord. Par ce qu'il y a de plus sacré chez la femme, sa matrice, qui est son deuxième cerveau" (150-1). Ici, l'assimilation se fait en deux temps. Il y a le lavage de cerveau et l'ensemencement. Pour parachever la conversion et compléter un lavage de cerveau problématique, le Professeur va prendre possession du deuxième cerveau d'Orimita, à savoir sa matrice, sa mémoire organique. Cet ensemencement d'une matrice bosniaque musulmane par une semence serbe n'a pas lieu ici dans le contexte d'une mise en contact/ en relation de deux peuples qui donnerait vie à une nouvelle identité multiple et rhizomatique, mais il est effectué en vue d'assimiler l'Autre pour toujours ne faire qu'Un.

Tout au long de son calvaire à l'intérieur du camp serbe, Orimita force son cerveau à se remémorer et à réciter des vers de Mallarmé. Ces nombreuses bribes mallarméennes, disséminées dans le roman, ne sont qu'images de bloc de glace, de froid, de gel, de givre, d'hiver, de glaciers, de blancheur, de nuit de glaçons, de neiges cruelles. Le cerveau d'Orimita, torturé, "pris dans les glaces d'un lac hyperboréen" (8) nous rappelle que 
"contrairement à l'idée reçue [. . .], le désespoir n'est pas noir, il est blanc" (61). Le bloc de glace illustre bien la perception identitaire d'Orimita. Qu'est-ce qu'un bloc de glace sinon un élément liquide, fluide, éternellement en mouvement qui sous l'effet du gel se glace, se fige en un bloc compact. C'est lorsqu'elle se sent menacée par le programme de conversion serbe, qu'Orimita se réfugie de façon obsessionnelle dans une série d'évocations lancinantes de gel et de blocs de glace comme pour "geler", pour arrêter son identité bosniaque. C'est un gel péremptoire qui nie le caractère fluide et perpétuellement en mouvement (l'eau) d'une identité-relation. Déjà, à la première page du roman, Orimita "se voyait en rêve traverser les massacres intacte" (7, souligné par nous), comme si son identité était complète, achevée, donc inaltérable. Le rêve lui laisse ses illusions de perfection identitaire, la réalité, elle, les brisera. Elle se veut intacte maintenant et à jamais. Pourtant, "l'émiettement en cantons ethniques" (189) de la carte de son pays, divisée en taches de couleurs symbolisant chacune un groupe éthnique particulier, ne lui rappelle que trop son trouble identitaire: "Elle, Orimita, musulmane mâtinée croate, se trouvait écartelée entre les taches vertes et jaunes noyées dans de grandes taches roses. Elle ne voulait pas être écartelée" (189). Elle qui veut croire à une identité-racine et qui dit être née "de la neige, congelée là-haut dans les régions sidérales" (103) se retrouve face à un "moi" problématique. Elle rejette la notion d'une identité en construction, toujours différée. C'est pourquoi elle s'évertue à cristalliser (à geler) son identité qu'elle voudrait parfaite, c'est-à-dire achevée. L'intrusion de l'autre (l'élément étranger, serbe ici) dans sa propre identité (musulmane) la déstabilise et provoque en elle un sentiment d'aliénation. Le drame d'Orimita est total puisqu'en excluant la présence de l'Autre dans sa propre identité, elle s'exclut elle-même. Son être, ou plutôt son étant n'est-il pas déjà dispersé dans l'étendue de la Relation? Son existence, c'est-à-dire sa venue au monde, n'a-t-elle pas déjà brisé la linéarité d'une filiation en ligne directe? Elle est née de père musulman et de mère croate. À l'instar des Serbes qui investissent 1 'homme du pouvoir exclusif de garantir une filiation, Orimita se considère musulmane et revendique cette identité patrilinéaire. Pourtant, son identité - qu'elle n'a jamais interrogée lors de son enfermement dans le camp serbe où elle se pensait en terme de différence, c'est-à-dire de "musulman" - devient trouble lorsque, libérée, elle se retrouve au milieu de ses camarades musulmans. "Elle sentait qu'elle se dédoublait. Orimita considérait Orimita" (194). L'identité individuelle d'Orimita (identité en émergence malgré elle) est confrontée à une identité collective "cristallisée" lorsqu'elle arrive à la Grande Mosquée, et qu'elle 
ne peut trouver sa place dans la foule musulmane où "des groupes compacts la bousculaient et la rejetaient" (215). Elle est donc exclue par cette identité "compacte" et "parfaite" qu'elle revendique et au nom de laquelle elle-même exclut.

Le caractère double de cette exclusion est inscrit tout entier dans le titre du roman. L'auteur offre sans choisir deux fins: la mort ou le meutre. Dans l'avant-dernier chapitre, Orimita se jette dans un brasier pour sauver un taureau appartenant à un vieux couple de paysans. Cette fin s'apparente à une mort volontaire qu'elle anticipe comme un soulagement. Dans le dernier chapitre, elle tue cinq Serbes avec un fusil acheté à un Moudjahidine, puis s'en va faire ses préparatifs en vue d'un très prochain voyage d'études à Paris. Orimita ne peut embrasser le Divers, elle ne peut fonctionner et se penser qu'à travers le prisme de l'exclusion et de l'identité unique et finie. Soit elle se nie (par la mort volontaire), soit elle nie l'autre (par le meurtre). Qu'elle fasse taire sa voix ou celle de l'autre, qu'elle nie sa singularité ou celle de l'autre, c'est la même chose, c'est la relation qu'elle tue. Cette naissance rétro-active promise par le Professeur, cette filiation recommencée ailleurs n'aura pas lieu. Orimita rétablit la linéarité de sa filiation en y effaçant toute intrusion de sang serbe lorsque sa matrice expulse un mort-né. L'exclusion de l'Autre est à l'oeuvre aussi bien dans le corps que dans l'esprit d'Orimita. Le Professeur avait choisi Orimita et ses camarades prisonnières pour son programme purificateur en raison de leur culture européenne: "Et comme vous avez la culture, je vais vous donner la naissance" (43), leur déclare-t-il. Le Professeur n'avait choisi, pour son programme de conversion, que des jeunes femmes musulmanes éduquées, notamment en littérature et culture française. Ainsi, considère-t-il sa mission à moitié accomplie en ce sens que leur esprit est déjà "occidentalisé" par la culture qu'elles ont reçu grâce à laquelle elles sont déjà (toujours selon le Professeur) "virtuellement revenues à [leur] origine naturelle, qui est slave et orthodoxe" (43): "Vos pensées, vos réflexions, que sais-je, vos rêves nocturnes, vous renvoient à Voltaire ou à Mallarmé, à Chaucer, Schiller, Cervantes, à Dante, au délicieux Jules Laforgue. Et non point à Mahomet, n'est-ce pas? (léger rire)" (42).

Tout au long de son internement, et de manière obsessionnelle et exclusive,

Orimita oblige sa mémoire défaillante (gelée) à réciter des vers de Mallarmé. Mais, le jour où elle essaie de se remémorer les mots d'un poète croate, elle se retrouve confrontée à l'impossibilité de faire co-exister dans son esprit - la culture croate et la culture française. Ainsi, cherche-telle à se "libérer de Mallarmé" (152), tel un "gros kyste dans son cerveau abominablement" (167). "Mallarmé la brûlait, c'était sa fièvre, sa blessure 
à elle" (181). Ce cerveau gelé ne peut embrasser la poésie croate qu'au prix de la négation de Mallarmé: "Et avec ce poème, qu'elle avait appris à l'école il y avait bien longtemps, elle eut la certitude qu'elle était en train d'expulser Mallarmé, dans la joie et dans la douleur" (203). Elle expulsera Mallarmé comme elle a expulsé son enfant mort-né innommable (puisque étranger à elle), cette "chose qu'elle sentait venir" (160) et que son corps (à l'instar de son esprit) n'avait pu concevoir. Lorsqu'Orimita enfante dans la joie et la douleur, ce n'est pas pour donner la vie mais la mort, pour se débarrasser de ce qui éclate et diffracte son identité-racine, pour résister à toute altération du moi. Afin de dépasser le caractère réducteur d'une construction identitaire qui relèverait du principe de référence à un modèle, il serait moins stérile de se définir par un "processus de singularisation" pour.reprendre la formule de Félix Guattari. De sorte que, si affirmer son identité "ne saurait avoir d'autre forme que narrative, car se définir c'est toujours en fin de compte se raconter"7, alors il faudrait se raconter (appréhender son identité) à n-1, c'est-à-dire en soustrayant sa singularité de la relation, de la totalité rhizomatique, sans exclure l'Autre qui est partie constituante du "Je" qui se construit, de cette identité toujours en devenir.

University of Kansas 


\section{Notes}

1. Janine Matillon, Les deux fins d'Orimita Karabegovic (Éditions Maurice Nadeau, 1996). Toutes mes références sont à cette édition et j'indiquerai dans le texte les pages auxquelles elles renvoient.

2. Édouard Glissant, Poétique de la Relation (Paris: Gallimard, 1990).

3. Édouard Glissant, Poétique de la Relation (Paris: Gallimard, 1990) 158

4. Édouard Glissant, Poétique de la Relation (Paris: Gallimard, 1990)25

5. Édouard Glissant, Poétique de la Relation (Paris: Gallimard, 1990)166.

6. Gilles Deleuze et Félix Guattari, Mille Plateaux (Paris: Minuit,1980) 13.

7. Voir, Paul Ricoeur, Temps et récit (Paris: Seuil, 1985). 


\section{Ouvrages cités}

Matillon, Janine. Les deux fins d'Orimita Karabegovic. Éditions Maurice Nadeau, 1996.

Glissant, Édouard. Poétique de la Relation. Paris: Gillimard, 1990. Deleuze, Gilles et Guattari, Félix. Mille Plateaux. Paris: Minuit, 1980. Ricoeur, Paul. Temps et récit. Paris: Seuil, 1985. 
